Research Article

\title{
Rapid Detection of Kaempferol Using Surface Molecularly Imprinted Mesoporous Molecular Sieves Embedded with Carbon Dots
}

\author{
Yu He, ${ }^{1}$ Junping Wang $\mathbb{D}^{1},{ }^{1}$ and Shuo Wang ${ }^{2}$ \\ ${ }^{1}$ State Key Laboratory of Food Nutrition and Safety, Tianjin University of Science and Technology, Tianjin 300457, China \\ ${ }^{2}$ Medical College, Nankai University, Tianjin 300350, China \\ Correspondence should be addressed to Junping Wang; potatowjp@163.com
}

Received 20 November 2019; Accepted 11 March 2020; Published 10 July 2020

Academic Editor: Eladia M. Pena-Mendez

Copyright (c) $2020 \mathrm{Yu} \mathrm{He}$ et al. This is an open access article distributed under the Creative Commons Attribution License, which permits unrestricted use, distribution, and reproduction in any medium, provided the original work is properly cited.

\begin{abstract}
This work demonstrates rapid sensing of kaempferol using active sensing material synthesized using the one-pot surface-imprinting synthesis method. This sensor consisted of molecularly imprinted polymer (MIP) consisting of mesoporous molecular sieves (SBA-15) loaded with carbon dots (CDs). Fourier transform infrared (FT-IR) spectroscopy confirmed successful incorporation of CDs onto the surface of imprinted mesoporous molecular sieves. Ordered hexagonal arrays of CDs@SBA-15@MIP mesopore structure were confirmed with transmission electron microscopy. Fluorescence intensity of CDs@SBA-15@MIP composites linearly correlated with kaempferol content in the $0.05-2 \mathrm{mg} / \mathrm{L}$ range. Detection limit was $14 \mu \mathrm{g} / \mathrm{L}$. MIPs were used for efficient detection of kaempferol in fruit and vegetable samples with recovery values from $80 \%$ to $112 \%$. The method has high sensitivity, low cost, good selectivity, and many application potentials useful for research and development of flavonoid monomer presence in food.
\end{abstract}

\section{Introduction}

Kaempferol, a polyphenolic antioxidant present in vegetables and fruits, is a very beneficial dietary component because it is able to reduce risk of cancer and various chronic illnesses [1]. Epidemiological studies showed direct correlation between amount of consumed kaempferol and cancer reduction [2]. Kaempferol helps to fight cancer and chronic diseases by boosting antioxidant-based defense of the organisms against aggressive and cancer-causing free radicals, which, at molecular level, translates into kaempferol control of key factors of cellular signal transduction routes linked to apoptosis, inflammation, angiogenesis, and metastasis [3, 4].

Most common kaempferol detection methods are high-performance liquid chromatography (HPLC) combined with UV-detection, mass spectrometry, colorimetry, etc [5-10]. Chromatography is accurate for detection of single or multiple components. However, it requires complex and tedious preprocessing steps and expensive equipment. Colorimetric methods are fast and easy and are the most common detection methods. Yet, they only detect total flavonoid contents. A technique to specifically and selectively detect kaempferol in food sample as well as to distinguish kaempferol from other flavonoids is urgently needed to screen and describe food nutritional contents. Such technique also needs to be very sensitive to variety of kaempferol contents as well as to be simple, inexpensive, and convenient. Previous work has highlighted the excellent potential for the application of fluorescence spectroscopy to food safety evaluations; as such, an approach can be employed both effectively in research contexts and in assessments of food quality [11]. When assessing food quality, this approach can be used to monitor the egg freshness during storage, to explore the evolution of extra virgin olive oils under illumination, to detect aflatoxin and related secondary metabolites, and to measure total amino acids in herbicide-stressed oilseed rape leaves [12-15]. 
At present, nanotechnology has facilitated the development of sensors with improved sensitivity and selectivity, thus offering novel opportunities for substantial innovation [16]. Two materials, used in this work to develop such technique, are mesoporous molecular sieves and carbon dots (CDs). Mesoporous molecular sieves are excellent extraction materials because of their ordered structures, high surface areas, uniform and adjustable pore sizes, and outstanding chemical stability $[17,18]$. CDs-based composites are relatively recently developed fluorescent materials, which demonstrate better photostability, brighter photoluminescence, better biocompatibility, and lower background noise in comparison to traditional organic dyes [19].

In this work, using these two materials, we created molecular imprinted polymers (MIPs) with the goal to obtain an effective and simple one-stage technique of material preparation for sensing. We created MIP based on mesoporous molecular sieve (SBA-15), which was loaded with CDs. Our main aim was to create a sensor capable to detect kaempferol accurately as well as with high sensitivity and selectively (Scheme 1). Surface molecular imprinting technique was used to obtain MIP composite. The resulting CDs@SBA-15@MIP composites consisted of highly ordered hexagonal mesopore arrays aligned as one-dimensional channels. At optimized conditions, linear correlation between sensor response and kaempferol concentration in the $0.05-2.0 \mathrm{mg} / \mathrm{L}$ range was observed. In this kaempferol concentration range, the sensor demonstrated rapid response time and excellent selectivity to distinguish kaempferol from its structural analogues. Fluorescencebased detection of kaempferol using CDs@SBA-15@MIPs as active sensing material demonstrates novel strategy for selective and sensitive kaempferol analysis in vegetable and fruits without need of bulky and expensive equipment such as liquid chromatography and mass spectrometry.

\section{Materials and Methods}

2.1. Materials. $\mathrm{N}$-( $\beta$-aminoethyl)- $\gamma$-aminopropyl methyldimethoxysilane (AEAPMS), 3-methacryloxypropyltrimethoxysilane (MPS), and 2, 2-azobisisobutyronitrile $(A I B N)$ were purchased from Tianjin Kermel Chemical Reagent (China). SBA-15 was obtained from Xfnano Reagents (Nanjing, China). Kaempferol was acquired from TCI Development (Shanghai, China). Citric acid (CA), acrylamide (AM), tetra-ethoxy-silane (TEOS), ethylene glycol dimethacrylate (EGDMA), and other chemicals were obtained from Sinopharm Chemical Reagent (Tianjin, China). Fruits and vegetables were purchased from a local grocery store.

2.2. Characterization. Fourier transform infrared (FT-IR) spectroscopy was performed using VECTOR-22 (Bruker, Germany). Fluorescence (FL) spectra were recorded using Thermo Scientific Lumina FL-4500 spectrometer (Thermo America, USA) with the $365 \mathrm{~nm}$ wavelength, $10 \mathrm{~nm}$ wide excitation, and emission slits as well as using $700 \mathrm{~V}$ photomultiplier tube voltage. Absorbance was measured using Thermo Scientific Evolution 300 UV-vis spectrophotometer (USA).
2.3. Carbon Dot Synthesis. CDs were obtained by a one-step reaction synthesis first implemented by Wang et al. [20]. For this purpose, $10 \mathrm{~mL}$ of AEAPMS was degassed by flushing the $100 \mathrm{~mL}$ three-necked flask with nitrogen gas for 5 minutes, after which the reaction solution was heated to $240^{\circ} \mathrm{C}$ and $0.5 \mathrm{~g}$ of CA was quickly added under constant vigorous stirring for 1 minute. After that, the system was allowed to cool naturally. Final CDs-containing products were collected by petroleum-ether-assisted precipitation performed 3 times. Samples were stored at $4^{\circ} \mathrm{C}$ until they were used and/or analyzed.

2.4. SBA-15-MPS Synthesis. SBA-15-MPS was fabricated using method reported by $\mathrm{He}$ et al. [21]. For this purpose, $500 \mathrm{mg}$ of SBA-15 was mixed with $50 \mathrm{~mL}$ of toluene. Then, $10 \mathrm{~mL}$ of MPS was added, and the mixture was stirred at $55^{\circ} \mathrm{C}$ for $24 \mathrm{~h}$ under nitrogen gas atmosphere. Final solid product formed was centrifuged, rinsed with toluene and methanol, and then dried at $45^{\circ} \mathrm{C}$ in a vacuum furnace for $24 \mathrm{~h}$.

2.5. Synthesis of CDs@SBA-15@MIPs and CDs@SBA-15@ NIPs. $0.28 \mathrm{mmol}$ kaempferol and $1.55 \mathrm{mmol}$ AM were dispersed in $25 \mathrm{ml}$ of tetrahydrofuran/ethanol mixture (2.5: 1 volume ratio) under constant stirring for $4 \mathrm{~h}$ at room temperature. The resulting suspension was named "mixture A." $5.7 \mathrm{mmol}$ of EGDMA, acting as a cross linker, $70 \mathrm{mg}$ of AIBN initiator, and $0.1 \mathrm{~g}$ of SBA-15-MPS were dispersed in $25 \mathrm{~mL}$ of tetrahydrofuran/ethanol mixture (with $3: 2$ volume ratio). This suspension was named "mixture B."

Mixture B was transferred into mixture A, and $20 \mu \mathrm{L}$ of CDs was added in A. The resulting solution was first stirred for $10 \mathrm{~min}$ and then flushed with nitrogen gas for $15 \mathrm{~min}$. To allow polymerization to proceed, the reaction vessel was sealed and kept for 24 hours at $60^{\circ} \mathrm{C}$ in a water bath, after which the final product was washed with methanol/acetic acid mixture (with 9:1 volume ratio). To ensure complete removal of the template, discarded liquid was analyzed by UV-vis spectroscopy (Scheme 1). Thus, rinsing of the final product stopped when no template was detected in the decanted solution. Final CDs@SBA-15@MIPs were dried at $60^{\circ} \mathrm{C}$ in vacuum for $10 \mathrm{~h}$. For comparison, CDs@SBA-15@ NIP was synthesized following the same route as described above without addition of kaempferol.

2.6. Measurements of FL. To evaluate adsorption and selectivity of the synthesized materials,1 mg CDs@SBA-15@ MIP or CDs@SBA-15@NIP was thoroughly dispersed in $4 \mathrm{~mL}$ of kaempferol (and its analogues) at different concentrations. The solutions were shaken for 2 hours at room temperature, and FL intensity of each solution was measured before and after its reaction with kaempferol.

2.7. Sample Preparation. Fruits and vegetables were crushed in a mixer grinder. $1 \mathrm{~g}$ of each sample was then placed into a $50 \mathrm{~mL}$ disposable screw-capped polypropylene tube. Then, $25 \mathrm{ml}$ of $80 \%$ ethanol was added for a 30 min extraction 


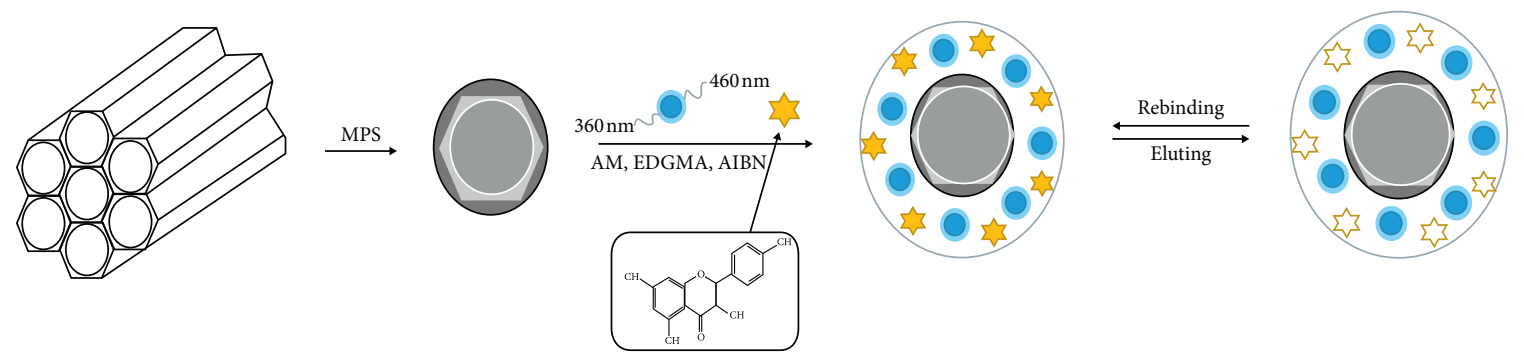

Scheme 1: Preparation of CDs@SBA-15@MIP.

process performed in an ultrasonic bath. Extraction step was repeated two times. The obtained filtrate was then evaporated using a rotary evaporator. The resulting solid residue was dissolved in $2 \mathrm{~mL}$ of ethanol and filtered through a nylon micropore membrane with $0.45 \mu \mathrm{m}$ micropores. Samples were stored in a clean glass bottle until further characterization.

2.8. HPLCAnalysis. Extracts were purified using $\mathrm{C}_{18}$ column to eliminate matrix interferences. Eluent obtained from the column was dried on a rotary evaporation, dissolved again in $2 \mathrm{~mL}$ of ethanol, and finally filtered using a nylon membrane with $0.45 \mu \mathrm{m}$ micropores for further HPLC-UV tests.

High-performance liquid chromatography (HPLC) coupled with UV was performed by using the LC-20AT (Shimadzu) chromatograph. Samples sizes were $20 \mu \mathrm{L}$. Analysis was performed using a diode array detector (SPD20A, Shimadzu) at $360 \mathrm{~nm}$. Mixture of water and methanol at $2: 3$ volume ratio was used as a mobile phase, flow rate of which was of $1.0 \mathrm{~mL} / \mathrm{min}$.

\section{Results and Discussion}

3.1. Properties of Obtained CDs@SBA-15@MIP. FT-IR analysis of CDs@SBA-15@MIP and CDs@SBA-15@NIP composites showed similar patterns with characteristics bands of MIPs and NIPs at 1658, 1255, and $1460 \mathrm{~cm}^{-1}$ (Figure 1). Thus, structures and compositions of CDs@SBA15@MIP and CDs@SBA-15@NIP were similar, and the only difference was that template molecules were extracted from MIPs during the imprinting process.

Figures 2(a) and 2(b) show SEM images exhibiting the surface morphology of CDs@SBA-15@MIP and CDs@SBA15@NIP. Both samples had a rough surface and a relatively narrow size distribution. The morphology of SBA-15 revealed via TEM indicated the presence of highly ordered hexagonal mesopore arrays aligned so as to form one-dimensional channels (Figure 2(c)).TEM of CDs@SBA-15@ MIP also revealed a well-ordered morphology, in which the one-dimensional mesopore structure of SBA-15 was still visible (Figure 2(d)).

CDs@SBA-15@MIP was then used for the detection of kaempferol as shown in Figure 3. Fluorescence spectrum of CDs@SBA-15@MIP significantly changed upon kaempferol presence in the matrix judging by its significantly decreased intensity (Figure 3(c)), which decreased more at higher kaempferol concentration (Figure 3(d)). After the template

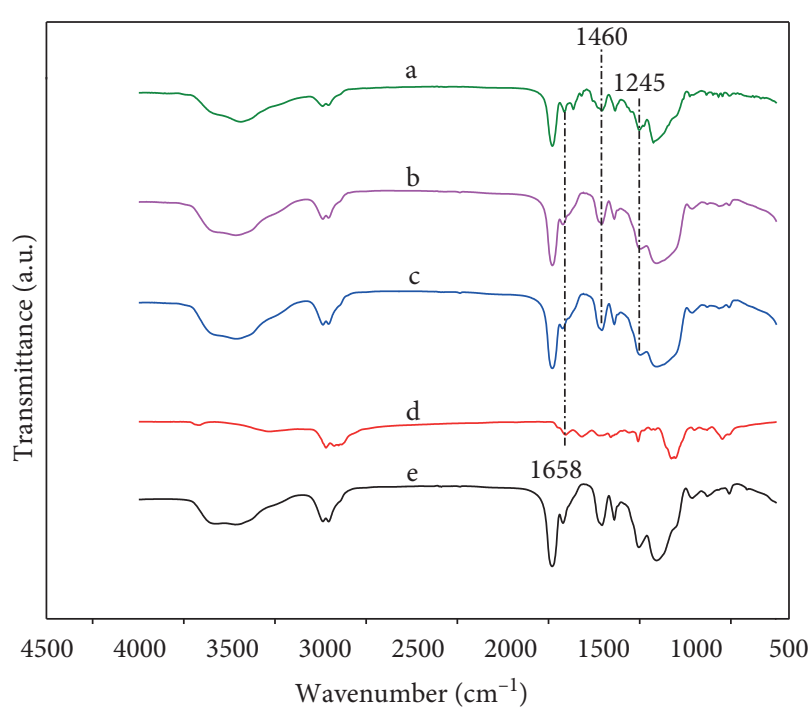

FIGURE 1: FT-IR spectra before (a) and after (b) extraction kaempferol of CDs@SBA-15@MIP.FT-IR spectra of CDs@SBA-15@ NIP after extraction (c) as well as of CDs (d) and kaempferol (e).

was extracted, fluorescence intensity of CDs@SBA-15@MIP (Figure 3(a)) became similar to the intensity of fluorescence of CDs@SBA-15@NIP (Figure 3(b)). Furthermore, the degree of quenching is affected by the concentration of kaempferol.

Fluorescence quenching upon kaempferol presence might be because of energy resonance transfer (FRET), which occurs between two fluorescent molecules in proximity to each other. UV-vis spectrum of kaempferol and CDs@SBA-15@MIP has similar absorption band gap values (Figures 4(a) and 4(b), respectively). Thus, energy resonance transfer would be easy between these two molecules. When the UV absorption peaks of kaempferol and FL peak of CDs@SBA-15@MIP overlap, nonradiative energy transfer occurs [22].

Analysis of adsorption kinetics helped us to evaluate FL quenching response rate of MIP. Fluorescence quenching value $\left(F_{0} / F\right)$ of the MIPs and NIPs was studied at different adsorption times. Sensor based on CDs@SBA-15@MIP showed higher fluorescence response to kaempferol than sensor based on CDs@SBA-15@NIP because of higher cavitation density and specific recognition sites (Figure 5). Adsorption of CDs@SBA-15@MIP was fast, and adsorption balance was achieved within 30 minutes. Therefore, $30 \mathrm{~min}$ was selected as the best detection time. Materials synthesized 


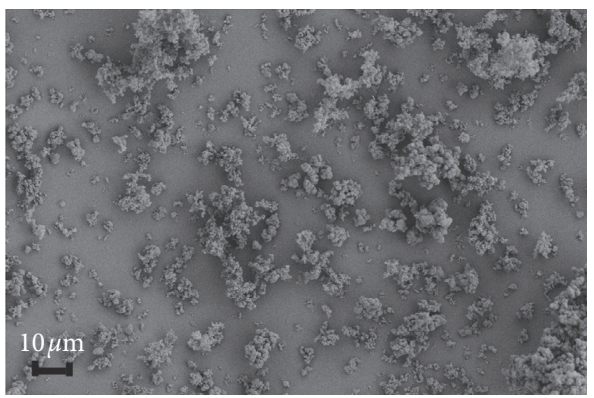

(a)

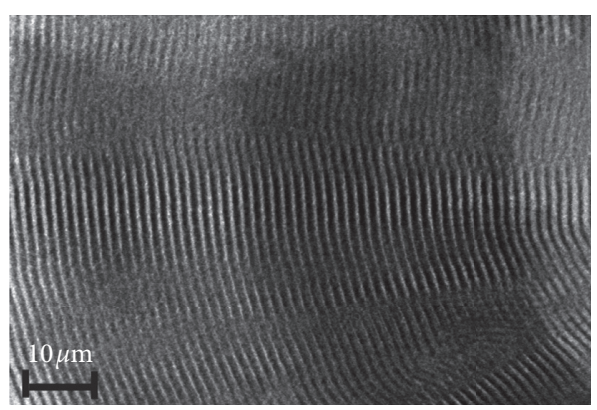

(c)

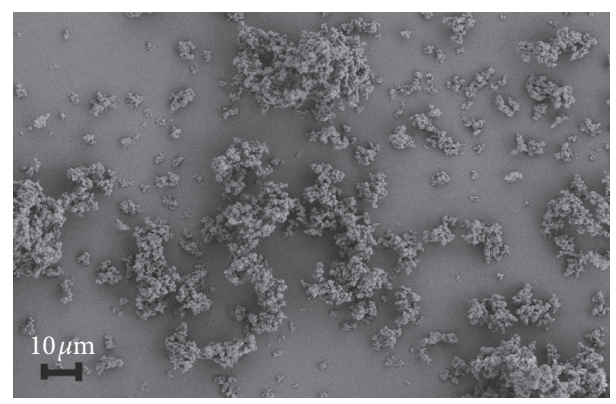

(b)

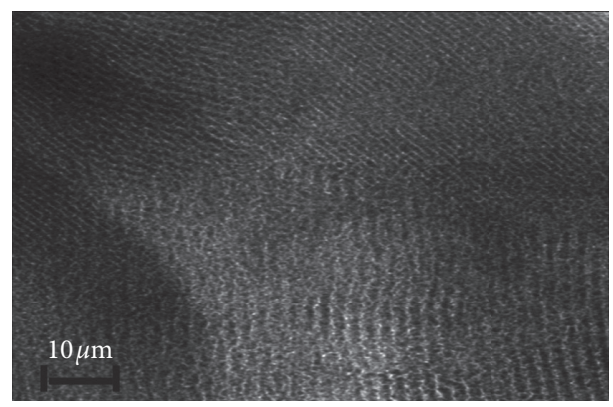

(d)

FIGURE 2: SEM micrographs images of (a) CDs@SBA-15-MIP and (b) CDs@SBA-15@NIP. TEM micrographs of (c) SBA-15 and (d) CDs@ SBA-15@MIP.

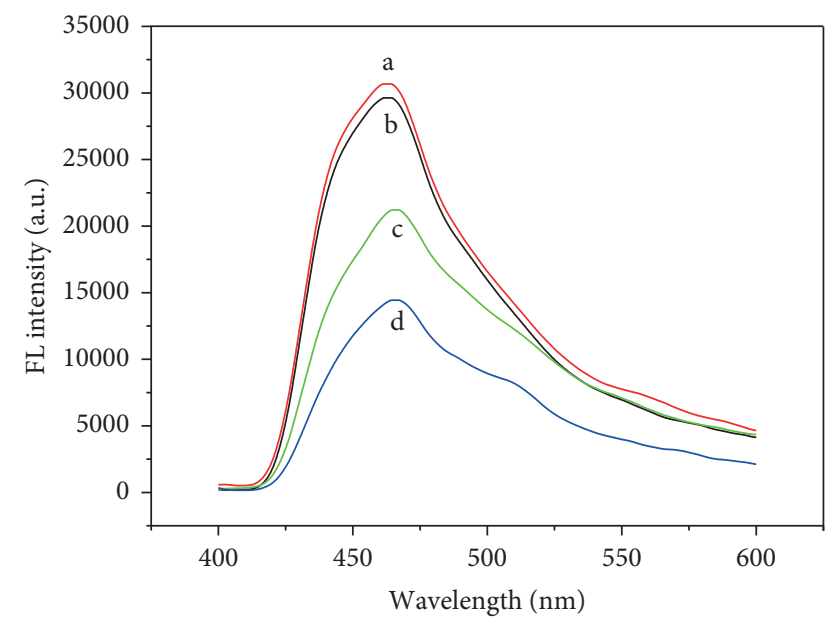

FIGURE 3: FL spectra of (a) CD @@SBA-15@MIP (after extraction), (b) CD $@$ SBA-15@NIP, (c) CD $@$ @SBA-15@MIP (before extraction), and (d) CDS@SBA-15@MIP in the presence of $1.0 \mathrm{mg} / \mathrm{L}$ of kaempferol.

by traditional bulk polymerization techniques usually demonstrate slower adsorption rates: sometimes up to 24 hours are needed to achieve equilibrium [23].

3.2. Specificity and Selectivity Experiments. Specificity and selectivity are the key characteristics demonstrating how successful molecular imprinted polymers are. Thus, we studied sensitivity of CDs@SBA-15@MIP relative to kaempferol and its structural analogues (such as myricetin (MYR), rutin (RT), and chlorogenic acid (CHA). FL

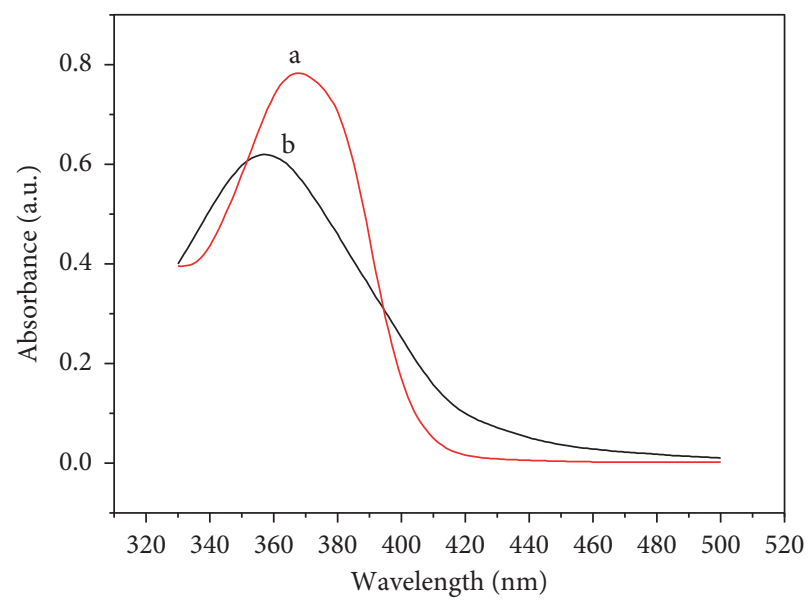

FIGURE 4: UV-vis spectra of kaempferol (a) and CDs@SBA-15@ MIP (b).

responses (presented as $F_{0} / F$ ) of CDs@SBA-15@MIP and CDs@SBA-15@NIP to kaempferol (KAE), MYR, RT, and CHA are shown in Figure 6. FL response of CDs@SBA-15@ MIP relative to $0.5 \mathrm{mg} / \mathrm{L}$ of kaempferol was much larger than for its structural analogues, which confirms better adsorption capacity. High specificity of CDs@SBA-15@MIP towards kaempferol was probably because shapes of MIP cavities fitted very well with kaempferol molecular structure. Other analogues did not bind strongly enough on the imprinted cavities.

Metal ions can also cause quenching of carbon point fluorescence. To investigate resistance of CDs@SBA-15@ MIP and CDs@SBA-15@NIP to interference from metal 


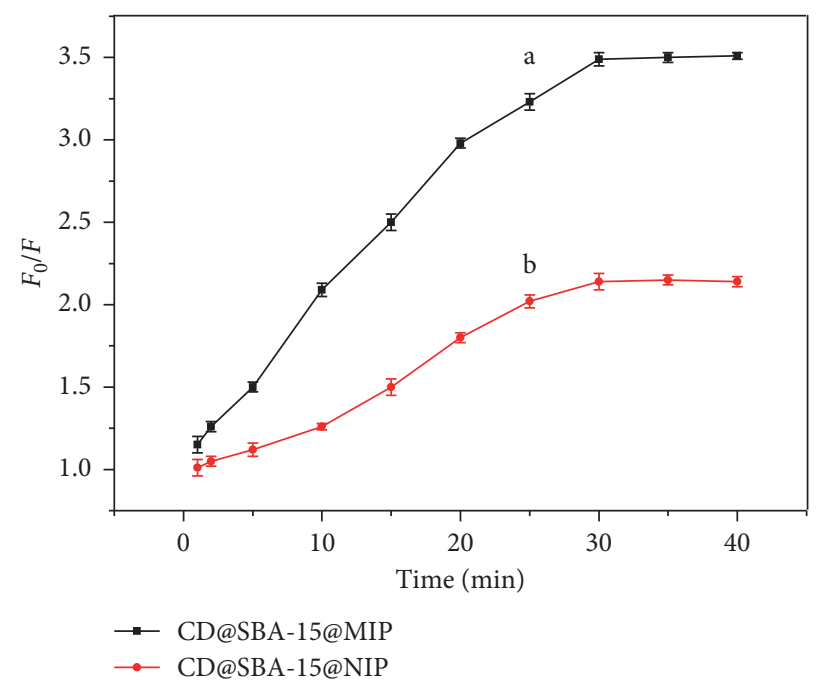

FIGURE 5: Kinetic uptake of kaempferol molecules by (a) CDs@SBA-15@MIP and (b) CDs@SBA-15@NIP.

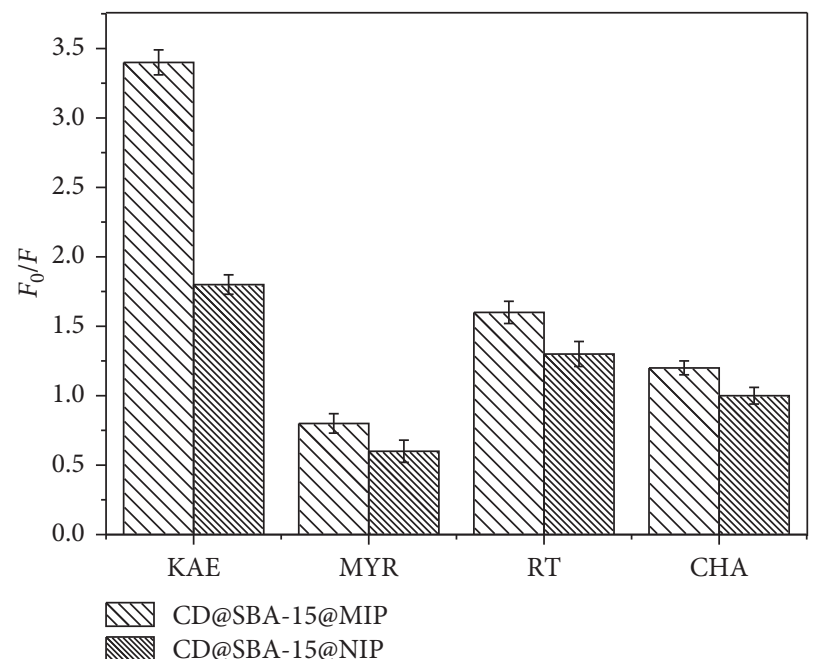

FiguRE 6: FL response of CDs@SBA-15@MIP and CDs@SBA-15@NIP towards kaempferol and its three structural analogues: CHA, MYR, and RT.

ions, fluorescence of CDs@SBA-15@MIP and CDs@SBA$15 @ N I P$ was measured under the presence of $\mathrm{Na}^{+}, \mathrm{Ca}^{2+}$, $\mathrm{Mg}^{2+}, \mathrm{Fe}^{3+}, \mathrm{Cu}^{2+}, \mathrm{Zn}^{2+}$, and $\mathrm{K}^{+}$. Binding capacity of CDs@ SBA-15@MIP and CDs@SBA-15@NIP towards kaempferol in the presence of different metal ions is shown in Figure 7(a). Figure 7(b) shows metal ions on CDs@SBA-15@ MIP and CDs@SBA-15@NIP.

These results illustrate that metal ions did not change FL signals of CDs@SBA-15@MIP and CDs@SBA-15@NIP under the kaempferol presence. Statistical analysis showed no signification difference between the groups $(p>0.05)$. Thus,CDs@SBA-15@MIP seems to be an ideal sensor for the highly selective detection of kaempferol.

3.3. Kaempferol Detection Using Calibration Curve. Changes in FL intensity of CDs@SBA-15@MIP and CDs@ SBA-15@NIP after adsorption of different concentrations of kaempferol are shown in Figure 8.
FL intensity decreased significantly as kaempferol concentration increased. FL quenching of any system typically follows the Stern-Volmer equation [24]:

$$
\frac{F_{0}}{F}=\operatorname{Ksv}[Q]+1 \text {, }
$$

where $F_{0}$ and $F$ are FL intensities in the absence and presence of kaempferol, respectively; Ksv is the Stern-Volmer constant, and $[Q]$ is the quencher concentration. We used this equation to quantify various quenching constants as well as Ksv ratios for MIP and NIP (Ksv-MIP/Ksv-NIP), which was used to define imprinting factor to determine selectivity.

A linear relationship between FL response and kaempferol concentration from 0.05 to $2.0 \mathrm{mg} / \mathrm{L}$ showed correlation coefficient equal to 0.9973 . The corresponding equation was as follows: $F_{0} / F=1.016 Q+1.056$.

Limit of detection (LOD) was $14 \mu \mathrm{g} / \mathrm{L}$ which is equal to $3 \sigma / k$, where $\sigma$ is standard deviation of the response and $k$ is the intercept of the calibration curve. 


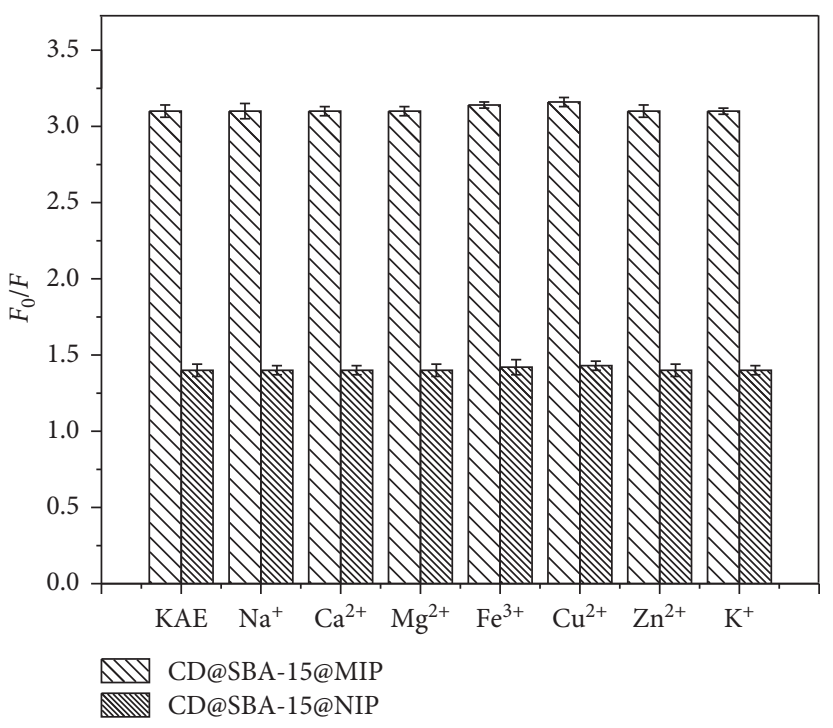

(a)

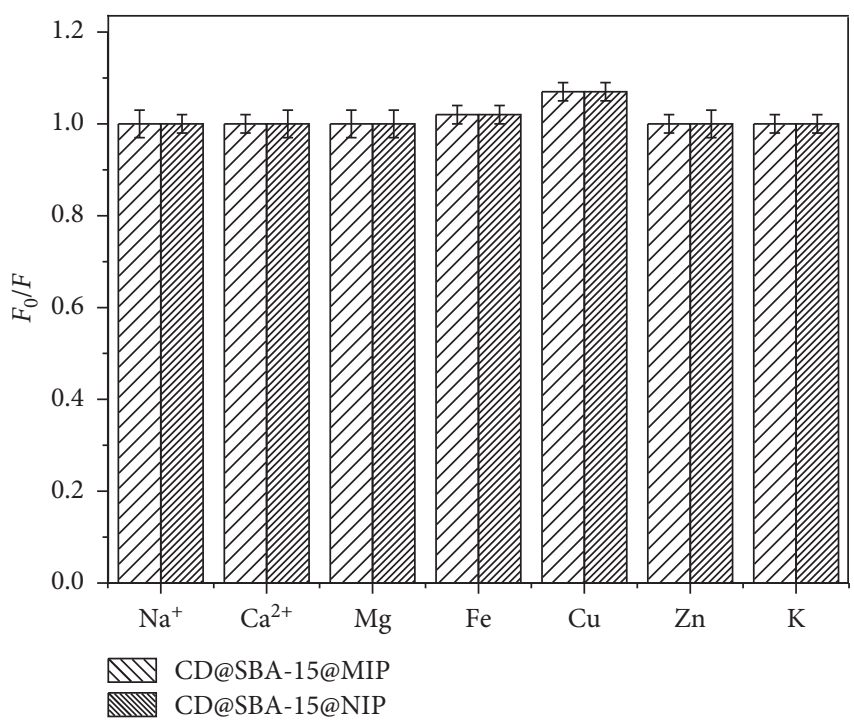

(b)

FIGURE 7: (a) Effect of the presence of metal ions on the recognition of kaempferol by CDs@SBA-15@MIP and CDs@SBA-15@NIP. (b) Identification of metal ions on CDs@SBA-15@MIP and CDs@SBA-15@NIP.

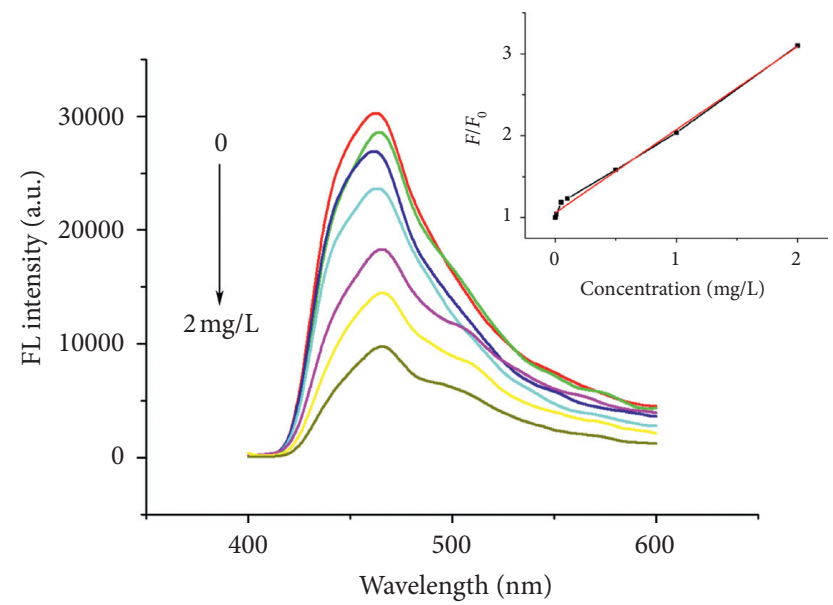

(a)

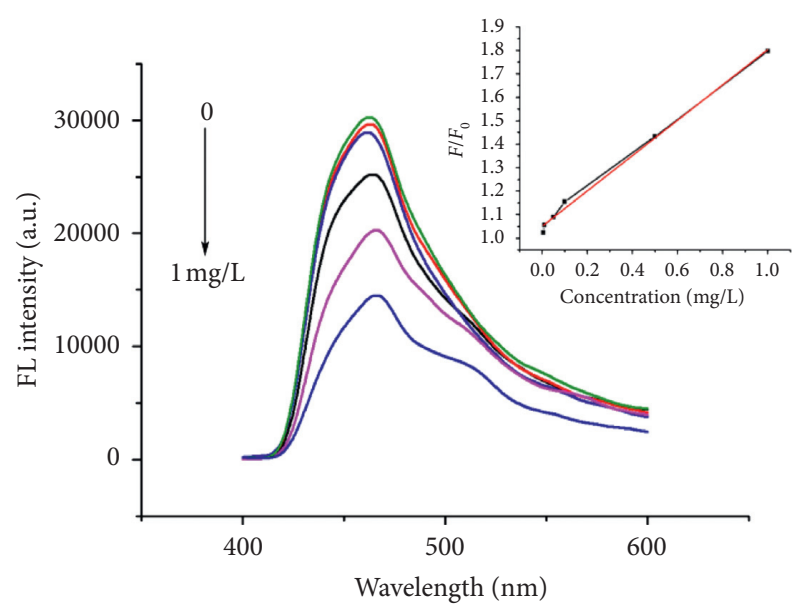

(b)

Figure 8: FL spectra of CDs@SBA-15@MIP (a) and CDs@SBA-15@NIP (b) in the presence of different kaempferol concentrations.

TABLE 1: Recovery data for kaempferol detection in various food samples obtained using CDs@SBA-15@MIPs and HPLC.

\begin{tabular}{|c|c|c|c|c|c|c|c|}
\hline \multirow{2}{*}{ Sample } & \multirow{2}{*}{ Added $\left(\mu \mathrm{g} \cdot \mathrm{kg}^{-1}\right)$} & \multicolumn{3}{|c|}{ FL } & \multicolumn{3}{|c|}{ HPLC } \\
\hline & & Found $\left(\mu \mathrm{g} \cdot \mathrm{kg}^{-1}\right)$ & Recovery $(\%, n=3)$ & RSD (\%) & Found $\left(\mu \mathrm{g} \cdot \mathrm{kg}^{-1}\right)$ & Recovery $(\%, n=3)$ & RSD (\%) \\
\hline \multirow{3}{*}{ Coriander } & 5 & 4.7 & 94 & 3.47 & 5.6 & 112 & 7.5 \\
\hline & 25 & 24.9 & 99.6 & 1.73 & 26.2 & 104.8 & 6.3 \\
\hline & 50 & 50.1 & 100.2 & 2.48 & 44.6 & 89.2 & 5.5 \\
\hline \multirow{3}{*}{ Strawberry } & 5 & 4.9 & 98 & 5.22 & 3.5 & 90 & 15.1 \\
\hline & 25 & 21.6 & 86.4 & 7.41 & 20 & 80 & 6.9 \\
\hline & 50 & 48.4 & 96.8 & 3.98 & 47.5 & 95 & 5.8 \\
\hline \multirow{3}{*}{ Orange } & 5 & 4.4 & 88 & 1.48 & 4.6 & 92 & 13.7 \\
\hline & 25 & 23.6 & 94.4 & 1.61 & 28.1 & 112.4 & 9.4 \\
\hline & 50 & 52.4 & 104.8 & 1.48 & 45.4 & 90.8 & 6.8 \\
\hline
\end{tabular}


TABle 1: Continued.

\begin{tabular}{|c|c|c|c|c|c|c|c|}
\hline \multirow{2}{*}{ Sample } & \multirow{2}{*}{ Added $\left(\mu \mathrm{g} \cdot \mathrm{kg}^{-1}\right)$} & \multicolumn{3}{|c|}{ FL } & \multicolumn{3}{|c|}{ HPLC } \\
\hline & & Found $\left(\mu \mathrm{g} \cdot \mathrm{kg}^{-1}\right)$ & Recovery $(\%, n=3)$ & RSD (\%) & Found $\left(\mu \mathrm{g} \cdot \mathrm{kg}^{-1}\right)$ & Recovery $(\%, n=3)$ & RSD (\%) \\
\hline \multirow{3}{*}{ Tomato } & 5 & 4.9 & 98 & 1.65 & 4.6 & 92 & 9.4 \\
\hline & 25 & 23.9 & 95.6 & 3.59 & 26.8 & 107.2 & 4.8 \\
\hline & 50 & 43.5 & 87 & 5.54 & 40.3 & 80.6 & 3.3 \\
\hline \multirow{3}{*}{ Carrot } & 5 & 4.6 & 92 & 1.91 & 4.4 & 88 & 13 \\
\hline & 25 & 24.2 & 96.8 & 2.32 & 23.4 & 93.6 & 5 \\
\hline & 50 & 51.4 & 102.8 & 2.8 & 44.2 & 88.4 & 6.7 \\
\hline \multirow{3}{*}{ Celery } & 5 & 4.4 & 88 & 3.57 & 4 & 80 & 12.8 \\
\hline & 25 & 23.8 & 95.2 & 4.3 & 25.8 & 103.2 & 10.4 \\
\hline & 50 & 45.9 & 91.8 & 7.34 & 46.9 & 93.8 & 5 \\
\hline \multirow{3}{*}{ Apple } & 5 & 4.3 & 86 & 2.3 & 3.7 & 82 & 12.7 \\
\hline & 25 & 23.2 & 92.8 & 3.71 & 18.4 & 84 & 8.9 \\
\hline & 50 & 49.4 & 98.8 & 2.2 & 49.7 & 99.4 & 6 \\
\hline
\end{tabular}

3.4. Kaempferol Detection in Fruits and Vegetables Using CDs@SBA-15@MIPs. To test practical application of our sensor, seven fruits and vegetables samples were selected. To ensure accuracy, we selected samples with low concentration of kaempferol for standard recovery experiment to eliminate matrix interference $[9,25]$. Three high concentrations of kaempferol were used to obtain sensor FL in order to later calculate its recovery rate. Recovery and RSD of kaempferol were $80-112 \%$ and $1.48-7.41 \%$, respectively. These values show feasibility of the kaempferol detection method in actual food samples. FL and HPLC results are shown in Table 1.

Detection results of CDs@SBA-15@MIP as a sensing material with fluorescence response in all seven food samples agreed very well with the HPLC results. Thus, the method developed in this work demonstrated high accuracy and can be used for practical applications because it satisfies detection requirements for the actual food samples. Detection procedure of this method was simple, accurate, and fast. Thus, it has a strong potential to be widely used in variety of practical applications.

\section{Conclusions}

Composites based on MIPs containing CDs-embedded SBA-15 were fabricated using a simple, one-stage, and onepot surface-imprinting synthesis technique. The resulting CDs@SBA-15@MIP material was used to fabricate sensor for fast and simple detection and concentration determination of kaempferol in food. SBA-15 used in this work had a well-defined mesoporous structure, which demonstrated excellent affinity and high capacity towards kaempferol as well as overall stability, all of which makes it an excellent adsorbent relative to kaempferol. Advantages of the sensor are reduced analysis time, excellent recovery, and repeatability, all of which eliminate complex multistep preparation of food samples required for equipment-intensive methods such as HPLC and MS. A method like ours offers rapid identification and quantification of a single component; thus, we strongly believe it has future in practical applications.

\section{Data Availability}

The data used to support the findings of this study are available from the corresponding author upon request.

\section{Conflicts of Interest}

The authors declare that they have no conflicts of interest.

\section{Authors' Contributions}

$\mathrm{Yu} \mathrm{He}$ conceived, designed, and completed experiments and wrote the manuscript. Junping Wang and Shuo Wang revised the article as tutors. All authors read and approved the manuscript.

\section{Acknowledgments}

The authors would like to thank National Key R\&D Program of China (grant no. 2016YFD0401202) and Special Project of Tianjin Innovation Platform (grant no. 17PTGCCX00230) for the financial support. The authors also thank LetPub (http://www.letpub.com) for its linguistic assistance during the preparation of this manuscript.

\section{References}

[1] V. Maren, E. Selma, M. F. Freda et al., "Mutual interaction of phenolic compounds and microbiota: metabolism of complex phenolic apigenin-C- and kaempherol-O-derivatives by human fecal samples," Journal of Agricultural and Food Chemistry, vol. 66, no. 2, pp. 485-497, 2018.

[2] Y. C. Allen and C. C. Yi, "A review of the dietary flavonoid, kaempherol on human health and cancer chemoprevention," Food Chemistry, vol. 138, no. 4, pp. 2099-2107, 2013.

[3] H. Luo, B.-H. Jiang, S. M. King, and Y. C. Chen, "Inhibition of cell growth and VEGF expression in ovarian cancer cells by flavonoids," Nutrition and Cancer, vol. 60, no. 6, pp. 800-809, 2008.

[4] R. Sonia, "Effects of dietary flavonoids on apoptotic pathways related to cancer chemoprevention," The Journal of Nutritional Biochemistry, vol. 18, no. 7, pp. 427-442, 2007.

[5] D. G. Watson and E. J. Oliveira, "Solid-phase extraction and gas chromatography-mass spectrometry determination of kaempferol and quercetin in human urine after consumption 
of Ginkgo biloba tablets," Journal of Chromatography B: Biomedical Sciences and Applications, vol. 723, no. 1-2, pp. 203-210, 1999.

[6] H. H. Sari, O. K, I. Sirpa, H. Marina, M. M. Hannu, and A. R. Torronen, "Content of the flavonols quercetin, myricetin, and campherol in 25 edible berries," Journal of Agricultural and Food Chemistry, vol. 47, no. 6, pp. 2274-2279, 1999.

[7] E. J. Kim, H. J. Lee, H. J. Kim et al., "Comparison of colorimetric methods for the determination of flavonoid in propolis extract products," Korean Journal for Food Science of Animal Resources, vol. 37, no. 6, pp. 918-921, 2005.

[8] M. M. Howard, D. M. Casandra, and R. B. Gary, "Kinetics method for the quantitation of anthocyanidins, flavonols, and flavones in foods," Journal of Agricultural and Food Chemistry, vol. 49, no. 6, pp. 2727-2732, 2009.

[9] J. Ulla, K. Pia, and L. Torben, "Quantitative analysis of flavonols, flavones, and flavanones in fruits, vegetables and beverages by high-performance liquid chromatography with photo-diode array and mass spectrometric detection," Journal of Chromatography A, vol. 799, no. 1-2, pp. 101-110, 1998.

[10] M. Raquel, L. E. José, M. Trujillo et al., "Determination of phenols, flavones, and lignans in virgin olive oils by solidphase extraction and high-performance liquid chromatography with diode array ultraviolet detection," Journal of Agricultural and Food Chemistry, vol. 49, no. 5, pp. 2185-2192, 2001.

[11] K. Romdhane and B. Christophe, "Fluorescence spectroscopy measurement for quality assessment of food systems-a review," Food and Bioprocess Technology, vol. 4, no. 3, pp. 364-386, 2011.

[12] K. Romdhane, B. Nicolaï, and J. De Baerdemaeker, "Monitoring the egg freshness during storage under modified atmosphere by fluorescence spectroscopy," Food and Bioprocess Technology, vol. 1, no. 4, pp. 346-356, 2008.

[13] H. S. Natalia, L. Lourdes, A. Faten, R. C. Teresa, and M. R. Jean, "Fast fluorescence spectroscopy methodology to monitor the evolution of extra virgin olive oils under illumination," Food and Bioprocess Technology, vol. 10, no. 5, pp. 949-961, 2017.

[14] H. Zuzana, Y. Haibo, K. Russell, B. Robert, C. Thomas, and B. Deepak, "Fluorescence excitation-emission features of aflatoxin and related secondary metabolites and their application for rapid detection of mycotoxins," Food and Bioprocess Technology, vol. 7, no. 4, pp. 1195-1201, 2014.

[15] F. Liu, Z. L. Jin, M. S. Naeem et al., "Applying near-infrared spectroscopy and chemometrics to determine total amino acids in herbicide-stressed oilseed rape leaves," Food and Bioprocess Technology, vol. 4, no. 7, pp. 1314-1321, 2011.

[16] N. Suresh and S. J. Digvir, "Nanotechnology for the food and bioprocessing industries," Food and Bioprocess Technology, vol. 4, no. 7, pp. 39-47, 2011.

[17] S.-H. Wu, C.-Y. Mou, and H.-P. Lin, "Synthesis of mesoporous silica nanoparticles," Chemical Society Reviews, vol. 42, no. 9, pp. 3862-3875, 2013.

[18] L. Shang, T. Bian, B. Zhang et al., "Graphene-supported ultrafine metal nanoparticles encapsulated by mesoporous silica: robust catalysts for oxidation and reduction reactions," Angewandte Chemie International Edition, vol. 53, no. 1, pp. 250-254, 2014.

[19] L. Xu, G. Fang, M. Pan, X. Wang, and S. Wang, "One-pot synthesis of carbon dots-embedded molecularly imprinted polymer for specific recognition of sterigmatocystin in grains," Biosensors and Bioelectronics, vol. 77, no. 15, pp. 950-956, 2016.

[20] F. Wang, X. Zheng, Z. Hao, Y. L. Chun, and G. Z. Ying, "Highly Luminescent organosilane-functionalized carbon dots," Advanced Functional Materials, vol. 21, no. 6, pp. 1027-1031, 2011.

[21] H. He, L. G. Xiao, Y. S. Li et al., "Molecular imprinted polymers based on SBA-15 for selective solid-phase extraction of baicalein from plasma samples," Analytical and Bioanalytical Chemistry, vol. 47, no. 2, pp. 509-519, 2015.

[22] Z. L. Li, F. Feng, C. P. Man et al., "Sensitive determination of kaempferol using carbon dots as a fluorescence probe," Talanta, vol. 144, no. 1, pp. 390-397, 2015.

[23] X. Liu, W. Zhang, and Z. Chen, "Preparation of a novel molecularly imprinted polymer for the highly selective extraction of baicalin," Journal of Separation Science, vol. 38, no. 24, pp. 4233-4239, 2015.

[24] X. Wang, L. Cao, F. Lu et al., "Photoinduced electron transfers with carbon dots," Chemical Communications, no. 25, pp. 3774-3776, 2009.

[25] S. Hiroyuki, H. Yoshinori, N. Satoshi, A. Hitoshi, and K. Kazuki, "Simultaneous determination of all polyphenols in vegetables, fruits, and teas," Journal of Agricultural and Food Chemistry, vol. 51, no. 3, pp. 571-581, 2003. 